\title{
Application of Three-dimensional Design Software in Transmission Lines
}

\author{
Kejian $\mathrm{Nie}^{1,{ }^{*}}$, Xinming $\mathrm{Yu}^{1}$, Yuanhao $\mathrm{Chen}^{1}$, Yinhe $\mathrm{Lin}^{2}$, Xin $\mathrm{Ye}^{2}$ and Ming $\mathrm{Xie}^{2}$ \\ ${ }^{1}$ State GRID Fujian Economic Research Institute, Fuzhou 350000, China \\ ${ }^{2}$ Fujian Yongfu Power Engineering Co., Ltd, Fuzhou 350000, China
}

\begin{abstract}
The digitization of power grid projects is a development requirement in the era of big data, as well as an inevitable requirement for the State Grid Corporation of China to build digital power grids and improve life-cycle management. Three-dimensional design establishes three-dimensional models of ground conductors, insulators, and fittings through line electrical majors, and completes line poles and towers in a three-dimensional scene; line structure majors should establish iron tower models and basic models; survey majors should conduct topography and landforms and ground objects to complete three-dimensional scene construction. It can make the circuit design results more intuitive, reduce the drawing work of repeated components, and improve the design efficiency.
\end{abstract}

\section{Introduction}

At present, digital technology is gradually integrated into the power grid design industry, and interactive collaborative design and three-dimensional design promote the continuous development of information technology for power grid survey and design. The application of three-dimensional digital technology to the design of transmission line engineering has become a hot topic of current research ${ }^{[1]}$.

Three-dimensional design is mainly through the efficient management of remote sensing images, basic geography, high-precision topography, power grid topics, line design results and other data to construct threedimensional visualization scenes of project sites and corridors, and the macroscopic display of the project corridors and the microscopic design of the line towers in combination, it provides project corridor feature collection, line optimization, line optimization, tower ranking optimization, three-dimensional space verification, tower insulator string, spacer rod, antivibration hammer, grounding device configuration, tower long and short legs and basic three-dimensional visualization configuration, and power transmission lines The three-dimensional display of elements (foundation, towers, ground conductors, fittings, insulators, intelligent devices, etc.), engineering material quantity statistics, drawing output of corresponding design results, and engineering digital handover and other service support ${ }^{[2]}$.

At present, the formation, circulation, storage, and use of 3D design files are all carried out in 3D design software. However, due to the different functions, configurations, and data formats of various 3D design platforms, cross-platform file transfer and sharing are very difficult ${ }^{[3]}$. The archiving of three-dimensional design documents mainly adopts the method of outputting two-dimensional graphics for archiving, and some companies engrave the final version of electronic files into CD-ROM for archiving.

Format and archive a browsable format file at the same time. For the subsequent use of very important three-dimensional model, data information, attribute information, association relationship data, etc., there is no archive, which brings great inconvenience to the reuse of design results. How to collect and archive threedimensional design documents, improve the reuse rate of design results, provide knowledge support for collaborative design, and provide high-quality services for project owners are major issues facing power engineering companies ${ }^{[4]}$.

At present, the mainstream power grid survey and design adopts CAD 2D design. Due to the limitation of design methods and the level of designers, more and more defects are exposed in the two-dimensional design. For example, it is difficult to fully reflect the actual situation on the spot, which is not conducive to the checking of the electrified distance; professional coordination is not enough, and professional collisions are prone to occur; multiple data input leads to repeated entry, repeated verification and drawing, etc.

Using the three-dimensional digital design platform, the design and construction tasks were completed efficiently and quickly. The case is based on the database, based on high-precision image data, and based on the parameterized transmission line model to achieve collaborative operation, and deep integration of surveying and mapping practice and design, effectively improving design efficiency ${ }^{[5]}$.

The three-dimensional digital software has a positive effect on design optimization. At the same time, it

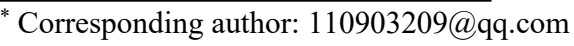


establishes geographic information models, ground object models, and 3D transmission line crossing simulation scenes, and truly restores the real-world model of overhead transmission lines, in order to optimize the route selection and the spatial distance of crossing ground objects.

\subsection{Model of conductor and ground wire}

In the 3D environment of the circuit design, the ground wire needs to select, calculate and draw the 3D effect of the wire through the electrical related calculation module, and establish the ground wire model database. The 3D model of the conductor and ground wire are shown in Figure 1 in the 3D scene of the transmission line.

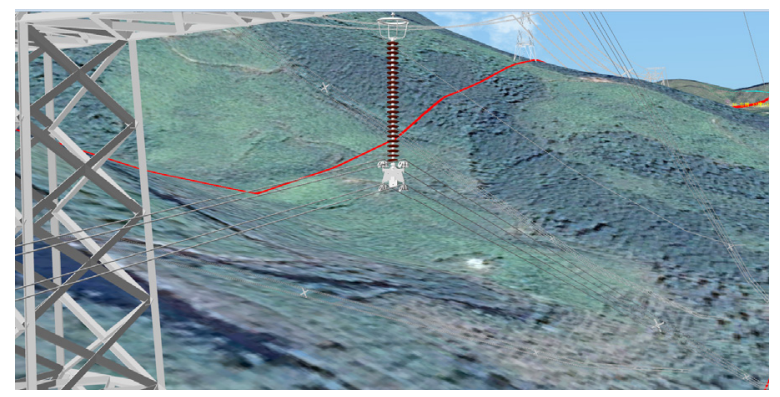

Fig. 1. The 3D model of the conductor and ground wire

\subsection{Model of insulator protective fitting and insulator string}

The system has a insulator protective fitting component data management module, which is mainly used to manage the insulator protective fittings component model, the insulator model, the insulator model and the insulator string model, and realize the functions of assembly and verification of the $3 \mathrm{D}$ model and the $3 \mathrm{D}$ model of the insulator protective fitting string.

After completing the assembly of the insulator protective fitting string, combined with the actual project using the number of insulators, the 3D model of the assembled insulator string is stored, and the string parameters and the effective hanging point coordinates of the selected string are entered at the same time. With the string function on the tower, the assembled insulator string can be selected for string matching. Show the 3D ranking effect in the ranking scene.

\subsection{Tower model}

The system realizes the analysis of the general data types of the poles and towers, completes the analysis to generate three-dimensional tower models with different call heights, and obtains the attribute data information such as the stress points of the towers. After completing part of the data entry, it is saved and put into the database. The data of the current pole tower model is put into the database to facilitate subsequent rankings, etc. Operation option is used.

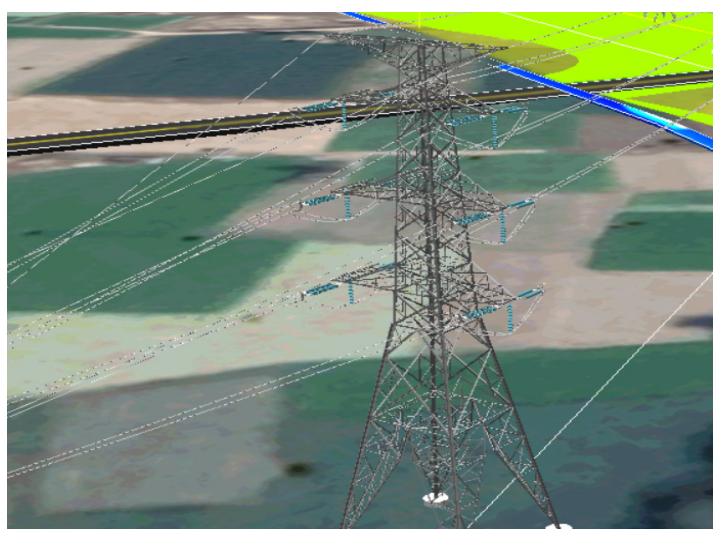

Fig. 2. Tower model in 3D scene

\subsection{Foundation model}

The basic data management of the system provides the parametric modeling and editing operations of the basic 3D model. The system provides a 3D model library for commonly used foundations, including pile foundations, rock foundations, and digged foundations.

\subsection{Route selection}

In the three-dimensional design of power transmission engineering, a full three-dimensional path design must be realized. The system is based on 2D and 3D GIS earth and massive geographic information data to realize path design, and can apply the design results to subsequent electrical and structural calculations.

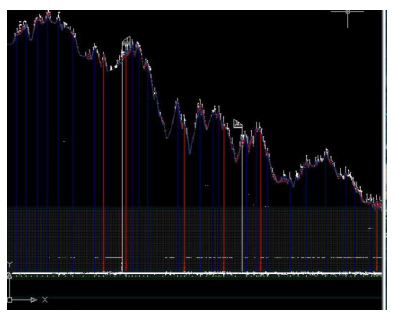

Fig. 3. Compare multi-scheme and selection

\subsection{The tower spotting in transmission line}

The towers arrangement of transmission lines is based on route selection, and arranging towers and related verifications on the existing optimal path. In addition, it can realize the functions of three-dimensional line roaming and ranking check.

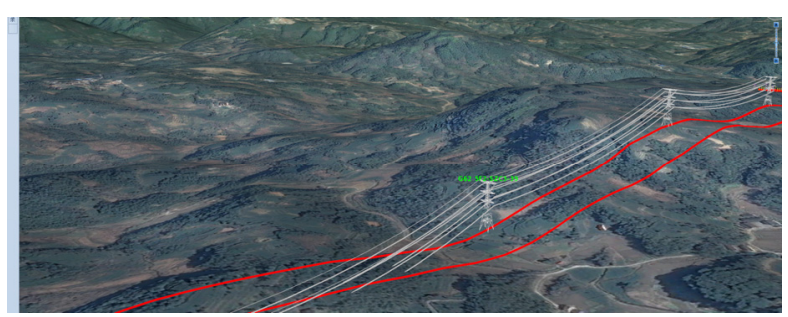

Fig. 4. The result of towers in $3 \mathrm{D}$ scene 


\section{Main results of applying 3D design}

The three-dimensional design system has the function of realizing automatic statistics of the number of poles and towers and the amount of materials, and can realize the one-click export of design drawings such as path diagrams, horizontal section diagrams, and mechanical characteristic curves (tables) of ground conductors, as well as tower coordinates and other design results. After arranging the towers, various space distances and distances between lines can be verified, and traces of verification can be kept.

\subsection{Automatic statistics of the number of poles and towers and the amount of materials}

After the line selection and tower arranging operations in the 3D design system, the number of poles and towers and the amount of related materials can be automatically generated.

\subsection{Automatic generation the line route map}

After the route of transmission lines plan is drawn, the route of transmission lines diagram can be automatically generated by the system. The path diagram export interface is as shown in the figure below.

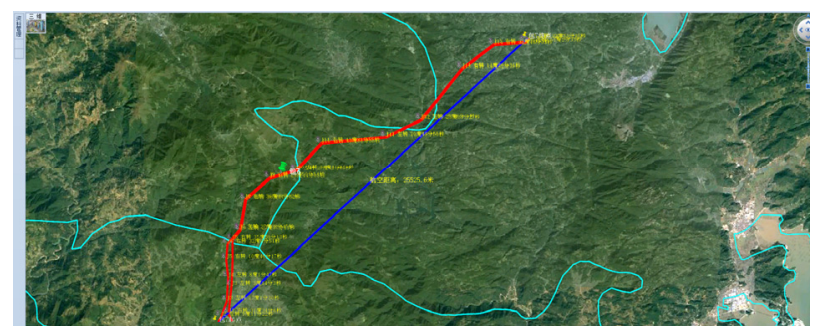

Fig. 5. Generate the route of transmission lines

\subsection{Automatic generation the plan and profile drawing}

Based on the existing line route, automatic generation the plan and profile drawing in the CAD window built into the system, as shown in the figure below. In the construction drawing stage, field survey data can also be imported to generate the horizontal section of the line in the construction drawing stage. And export the plan and profile drawing with dwg format.

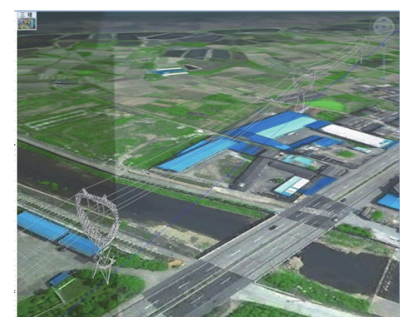

Fig. 6. The plan and profile drawing

\subsection{Automatic generation of the mechanical characteristic curve table of the conductor and ground wire}

After performing relevant calculations such as meteorological conditions, conductor and ground wire configuration in the system, a table of mechanical characteristics of the conductor ground wire can be automatically generated.

\subsection{Tower arrangement checking}

This function can realize the verification work in 5 aspects: conductor and ground wire, pole tower, insulator, cross-span distance and economic index. The conductor and ground wire verification includes: the safety factor verification of the lowest point of the conductor and ground wire sag, the safety factor verification of the conductor and ground wire suspension point, the everyday tension verification, the pull-up verification, the ground wire coordination verification, and the unbalanced tension Check. The tower verification includes four parts: the use of horizontal and vertical gear pitch verification, $\mathrm{Kv}$ value verification, and rotation angle verification and economic verification. Insulator string verification includes: insulation configuration verification, fittings front-end verification, first fittings matching verification, insulator string wind deflection angle verification, tensile string insulator string upside down verification, important crossover double string verification, Double string calibration on the outside of the corner tower of 45 degrees and above, double string calibration and $\mathrm{V}$ string calibration are hung on the outside of the corner tower with degrees and above. The crossing distance includes 5 parts: maximum sag check, maximum wind deviation check, cross angle check, parallel distance check, and safe distance check between poles and towers and ground and underground objects.

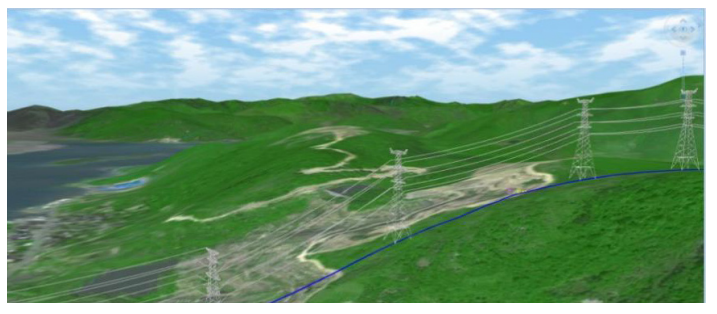

Fig.7. The horizontal span and vertical span checking

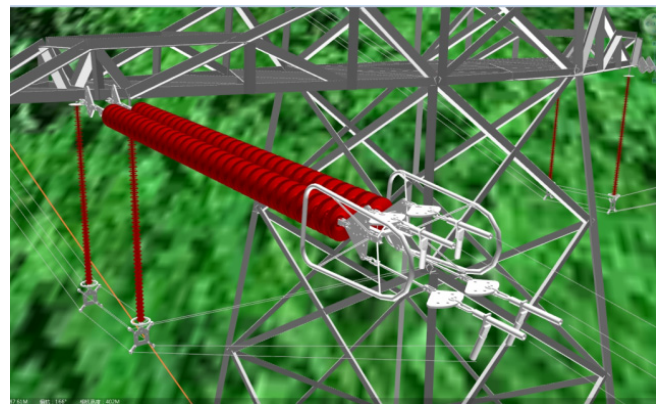

Fig.8.The distance between the tension tower jumper and the tower body checking 
Based on the three-dimensional platform, it can realize the function of fitting rotation and assembly. Take the porcelain insulator tensile string as an example, the three-dimensional model is established as shown in the figure.

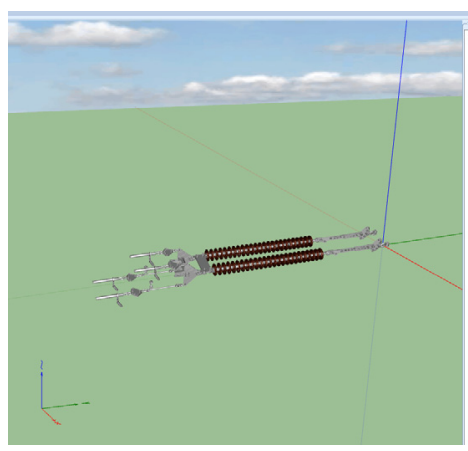

Fig.9.The fitting rotation

\subsection{The drawing of the refuge area}

Can draw the avoidance area and manage the avoidance area agreement file, realize the management and view of the agreement file and field measurement file in the $3 \mathrm{D}$ system.

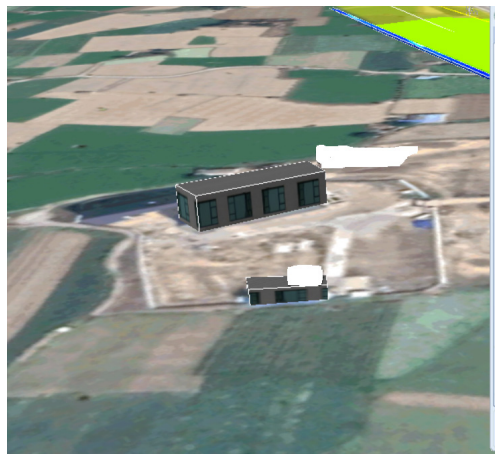

Fig.10.The refuge area

\subsection{Layer management}

The layer management function is mainly used to manage and display the images and vector layers in the system. According to the type of data, the layers are divided into two-dimensional layers and threedimensional layers.

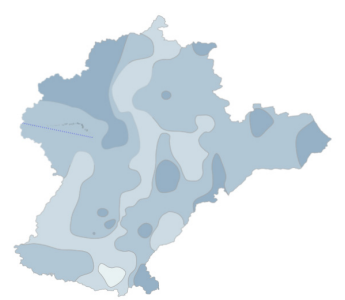

Fig.11.Wind zone vector

\section{Conclusion}

(1) By adopting the *.GIM output format of 3D design results, the model includes wires, ordinary ground wires, OPGW composite optical cables, online accessories (vibration hammers, spacers), towers, foundations and important crossovers; 3D design towers The 3D models of insulator strings and fittings adopt the models in the general model library to meet the minimum accuracy of the model and the requirements of coding rules.

(2) Based on the geographic information system, the three-dimensional model is used to preliminarily realize the comparison and selection of multiple paths, preliminary ranking, path optimization, clearance verification, selection of poles and towers, and basic selection.

(3) Based on the relevant data information in the line 3D design model, it can display the main channel cleaning, material and engineering quantity statistics.

(4) Three-dimensional visualization technology has very good application value for the development of the power industry. On the one hand, it can realize the display of the development results of the power industry. On the other hand, it can use the three-dimensional model to combine with the power object to realize the mutual correlation of parameters and attributes.

\section{Acknowledgments}

The project was funded by Consulting Specific Fund of the State Grid Fujian Electric Power Co., Ltd. (Fund Name: Deepen Application Research Technology of $110 \mathrm{kV}$ Pole and Tower Universal Design Module).

\section{References}

1. Interaction specification for $3 \mathrm{D}$ design model of power transmission and transformation engineering, Q/GDW 11809-2018.

2. Technical guidelines for three-dimensional design of power transmission and transformation engineering. Part 2: Overhead transmission lines, Q/GDW 11798.2-2018.

3. Specification for $3 \mathrm{D}$ design modeling of power transmission and transformation engineering. Part 2: Overhead transmission lines, Q/GDW 11810.22018.

4. Application Guidelines for Digital Design Coding in Transmission and Distribution Engineering Part 2: Line Engineering, Q/GDW11600.1-2016.

5. Technical guidelines for digital handover of power transmission and transformation engineering Part 2: Overhead transmission lines, Q/GDW 11812.22018 . 